\title{
Corrigendum: Decoding tumour phenotype by noninvasive imaging using a quantitative radiomics approach
}

Hugo J.W.L. Aerts, Emmanuel Rios Velazquez, Ralph T.H. Leijenaar, Chintan Parmar, Patrick Grossmann, Sara Carvalho, Johan Bussink, René Monshouwer, Benjamin Haibe-Kains, Derek Rietveld, Frank Hoebers, Michelle M. Rietbergen, C. René Leemans, Andre Dekker, John Quackenbush, Robert J. Gillies \& Philippe Lambin Nature Communications 5:4006 doi: 10.1038/ncomms5006 (2014); Published 3 Jun 2014; Updated 7 Aug 2014

The original version of this Article contained a typographical error in the spelling of the author Sara Carvalho, which was incorrectly given as Sara Cavalho. This has now been corrected in both the PDF and HTML versions of the Article. 